EPJ Web of Conferences 43, 01002 (2013)

DOI: $10.1051 /$ epjconf/20134301002

(C) Owned by the authors, published by EDP Sciences, 2013

\title{
Low-mass stars: Open problems all along their evolution
}

\author{
A. Weiss ${ }^{\mathrm{a}}$ and N. Heners \\ Max-Planck-Institut für Astrophysik, Karl-Schwarzschild-Str. 1, 85748 Garching, Germany
}

\begin{abstract}
Although low-mass stars have a comparatively simple structure, current stellar models are far from reproducing them accurately. In light of the observational progress, in particular of asteroseismology, the deficits of the theory of stellar structure and evolution become increasingly evident, and can no longer be hidden under the carpet of observational errors. In my brief review I will discuss a number of obvious problems of the models, which are mainly - and not surprisingly so-connected with convective and other mixing processes. They begin already on the pre-main sequence and continue throughout the complete evolution. In addition to the deficits in the treatment of physical processes I will also address our difficulties in obtaining completely satisfying agreement between different numerical codes. It will be shown that this concerns already the main sequence evolution of low-mass stars without a convective core. Keeping this in mind, it is no surprise that models for stars on the Asymptotic Giant Branch differ widely.
\end{abstract}

\section{INTRODUCTION}

Our understanding of the general evolution of low-mass stars (those with $M \lesssim 2.3 M_{\odot}$, the compositiondependent maximum mass for the ignition of helium under degenerate conditions) is fairly advanced. Thanks to improved computer codes we are able to follow their evolution from beginning to end. As an example, we show in Fig. 1 the evolution of a $1 M_{\odot}$ star of solar composition from the zero-age main sequence (ZAMS) to the white dwarf cooling track (the pre-main sequence phase was included in the calculation, but omitted in this figure, except for the very last phase of approaching the ZAMS). As the educated reader can easily verify, the model evolved up the red giant branch (RGB), ignited helium in the core flash (point D), moved to the clump (or horizontal branch) at an age of around 12.2 Gyr and from there up the asymptotic giant branch (AGB), where it experienced a few thermal pulses (TP), during which the mass loss increased drastically and removed close to $40 \%$ of the stellar mass. Finally, during a last TP, which can easily be recognized, the star left the AGB, crossed the Hertzsprung-Russell-diagram (HRD) in a few ten thousand years and began its final cooling to become a white dwarf. The complete evolution could be followed (with our own code GARSTEC [1]) in one computation, without any artificial intervention, e.g. to skip the core helium flash or the departure from the AGB. Therefore, one could state that we not only understand, but also can model the evolution of low-mass stars sufficiently well.

However, a closer look reveals that there are many problems all along such an evolution. Many of them are connected with convection, which we are far from having it understood completely. Much less we are able to model it appropriately. A recurring topic during this contribution will be overshooting. Additional mixing (or separation) processes such as atomic diffusion are neither treated properly. The core helium flash, although we can-technically-model it, may happen, at least in some aspects, quite differently from the hydrostatic models we are computing. Another deficit of the models is the unknown mechanism and even the amount of mass loss during the red giant phases. Do giants lose

\footnotetext{
ae-mail: aweiss@mpa-garching.mpg.de
}

This is an Open Access article distributed under the terms of the Creative Commons Attribution License 2.0, which permits unrestricted use, distribution, and reproduction in any medium, provided the original work is properly cited. 
Figure 1. Evolution of a $1 M_{\odot}$ star of solar composition from the zero-age main sequence (ZAMS) to the white dwarf cooling track. Tickmarks along the track indicate age, given also explicitly along the track in some cases. On the main-sequence age is marked in steps of $2 \mathrm{Gyr}$. Points A-D refer to the ZAMS, the turn-off (TO), the bottom of the red giant branch (RGB) and the ignition of helium.

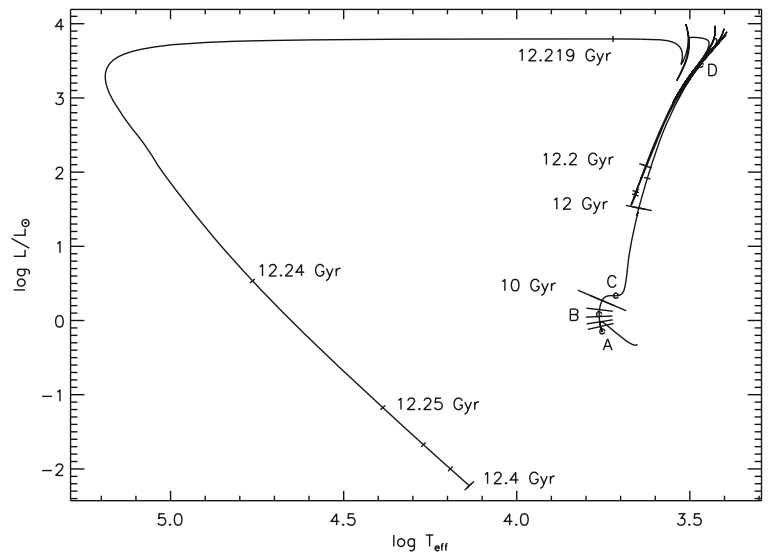

mass continuously, in recurring episodes, or even in a catastrophic event, for example, during the core helium flash? Why and by what degree differs the mass loss rate from star to star? Some of these aspects will be discussed in the following. I will not, however, deal with the general effect of rotation on the structure and evolution of low-mass stars, including the mixing processes resulting from rotation (see Eggenberger's contribution to this meeting). Neither will the uncertainties in the input physics, e.g. in opacities or nuclear reactions be the subject. However, I will also demonstrate that even for the simplest evolutionary phases models calculated with different codes differ appreciably.

\section{THE EARLY PHASES: PRE-MAIN SEQUENCE AND MAIN SEQUENCE}

\subsection{Lithium depletion}

The hydrostatic contraction phase on the pre-main sequence (PMS) is easily computed and in general it is assumed that whatever deficits the initial model should have, they would be forgotten when the model arrives on the ZAMS. However, ${ }^{7} \mathrm{Li}$ is a sensitive indicator of the temperature structure and the extension of (convectively) mixed regions in stars, and therefore the models have a memory beyond the pure thermal contraction timescale on the PMS. This is particularly important for any explanation of the solar lithium problem. Is the lithium deficit in the solar atmosphere by a factor of $\sim 160$ relative to the meteoritic value generated on the pre-MS or the MS? Published investigations reach from "overshooting from the convective envelope on the PMS is needed" [2] to the recognition that overshooting (OV) on the PMS leads to full depletion of $\mathrm{Li}$, which is not in agreement with the age-dependent Li-abundance in open clusters, and therefore accretion of Li during the MS would be needed [3].

Schlattl \& Weiss [4] have investigated in detail the effect of OV on Li depletion and on the sound speed profile in the solar case. Fig. 2 shows how $\mathrm{Li}$ is depleted under different assumptions for OV from the convective envelope during the PMS and/or MS, and how the transient convective core evolves if some OV from its boundary is assumed. If only the MS phase is considered (case 1; dashed line in both panels of Fig. 2), the solar Li problem can be solved for rather extensive OV (I omit the details here), but in this case the convective core persists until today, which is in clear contradiction to all seismic knowledge we have about the SUN. The same amount of OV employed on the PMS (case 1a; dot-dashed line in left panel) leads to complete Li-destruction long before the ZAMS. Intermediate OV (case 2; dash-dot-dot-dotted line in left panel) solves the Li problem nicely, but again contradicts helioseismology, since even smaller amounts of OV from the core (other dot-dashed lines in right panel) lead to a too long-existing convective core on the MS. The solid line in both panels are the standard case without OV that gives the best solar model. The conclusion therefore is that to solve the solar 

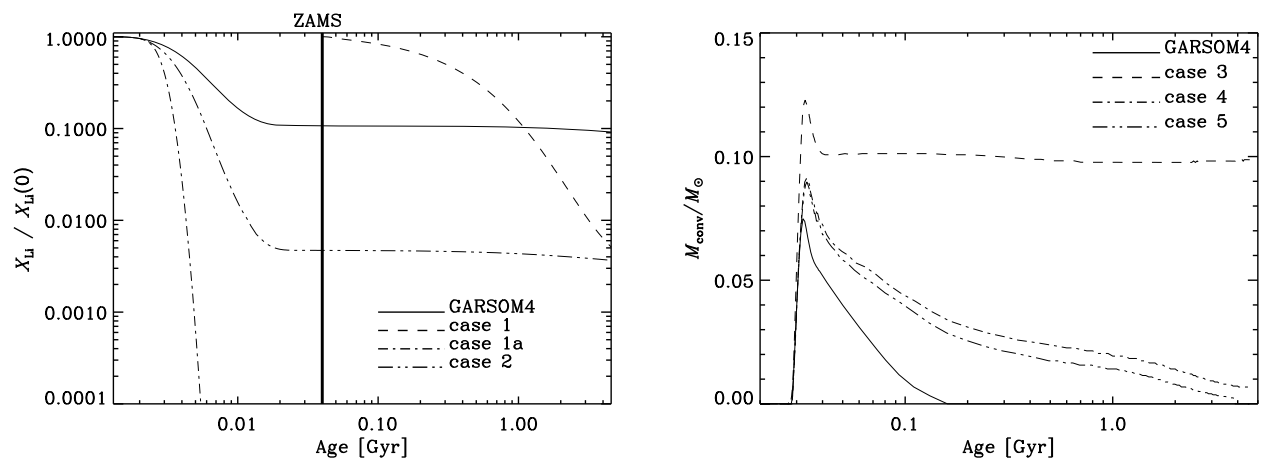

Figure 2. Left: ${ }^{7} \mathrm{Li}$ depletion on the pre-MS and MS for a solar model under various assumptions about convective overshooting. Right: the size of the convective core during the same phases (see text; from [4]).

Li-problem, overshooting must be fine-tuned, and be done so differently for core and envelope, and possibly even differently for the PMS and the MS phase. In other words, we have no clue if and how OV operates in the Sun!

But maybe the PMS of the Sun (and thus for other low-mass stars) is quite different from our simple models? Two examples will illustrate this. The first one is from a star formation simulation with a radiation-hydrodynamical code [5], which could follow the PMS evolution until an age of $1 \mathrm{Myr}$. At this point the protostar's surface was still about $500 \mathrm{~K}$ hotter than the standard hydrostatic stellar PMSmodels. Although it is unclear how much this also influences the stellar interior, whether it would solve the Li-problem, and for how long a different structure would persist, it illustrates how crude the standard PMS models might be.

The second example refers to the problem of the so-called "bright young Sun" (see [6] for a discussion). The existence of liquid water on Earth and Mars during the last $4 \mathrm{Gyr}$ cannot be simply due to a greenhouse effect [7], but may require an early solar luminosity about $20 \%$ higher than for a $1 M_{\odot}$ ZAMS star. This can be achieved by assuming an initial solar mass of about $1.07 M_{\odot}$ and a corresponding mass loss history. Indeed, if the latter is fine-tuned correctly, not only the "young bright Sun", but also the solar lithium problem could be solved, as well as the seismic problem that the new solar abundances [8] have constituted for the last 8 years!

\subsection{Overshooting from small convective cores}

While OV from the convective core of massive MS stars is a well-established fact, it is unclear how extensive it could be for low-mass stars in the transition mass range of $1.2 \lesssim M / M_{\odot} \lesssim 2.0$. While it is quite evident that it must be restricted (since the pressure scale-height $H_{P}$ diverges towards the centre and $\mathrm{OV}$ is in all standard treatments connected to $H_{P}$ ), it is unclear, by how much. The open cluster M67, at an age of about $4 \mathrm{Gyr}$, has been used [9] to investigate the existence of a convective core in the TOstars, in dependence of the old and new solar abundances. Including diffusion, both in the solar models (necessary for parameter calibration) and in the M67 models, which had been ignored in [9], it could be shown [10] that both solar abundance values lead to a satisfying match of the TO morphology, and thus that M67 is not really decisive, which of the two is to be preferred. It was also shown that the inclusion of a small amount of OV from the core of the TO stars improves the fit. The most interesting result, however, in the present context, concerns the fact that the use of an updated rate for the ${ }^{14} \mathrm{~N}(p, \gamma){ }^{15} \mathrm{O}$ reaction [11] requires core OV, otherwise the TO morphology could not be reproduced for neither of the two metallicities! Assuming, for the moment, that this rate would be wrong, this demonstrates how misleading a free choice of OV can be: it might repair model deficits that arise for completely different reasons! 
Figure 3. The Leiden 2009 model comparison for the "Giant Branches" workshop. Shown is the age-luminosity relation for models of $0.8 M_{\odot}(\mathrm{Z}=0.001)$. The models were computed with different codes (the solid line corresponds to the one by the author), but very similar physics input.

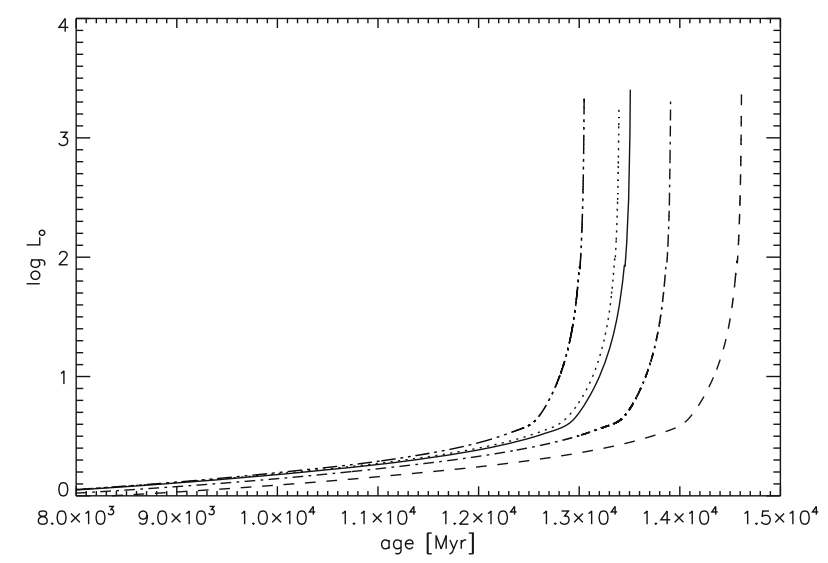

\subsection{Main-sequence lifetimes}

For the workshop "The Giant Branches" at the Lorentz Center in Leiden ${ }^{1}$ various groups provided stellar models for a model comparison. To our surprise the MS lifetimes for models with $M=0.8 M_{\odot}$ $(Z=0.001)$ and $M=1.1 M_{\odot}(Z=0.04)$ differed by up to $10 \%$, i.e. they ranged between 12.5 and $14.1 \mathrm{Gyr}$ (Fig. 3). Although in some cases better agreement with the input physics data (opacities, EOS, etc.) and more careful numerical computations reduced this difference, in a few cases it remained above the $5 \%$ limit without a clear reason being identified.

I close this section mentioning that the efficiency of diffusion, in particular that of gravitational sedimentation, which influences, among other things, also MS lifetime, luminosity, and effective temperature, is an open question as well. While the solar model requires it, it is disputed whether it is operating in low-metallicity stars of globular clusters.

\section{THE RED GIANT BRANCH}

Low-mass stars accumulate in a very narrow temperature range on the Red Giant Branch (RGB). The luminosity evolution depends primarily on the mass of the helium core and terminates at the RGB-tip when helium ignites in a violent off-center flash. The tip brightness is only weakly dependent on mass and composition. In spite of this apparent simplicity, the RGB evolution poses several open questions.

\subsection{Effective temperature and tip brightness}

It would be highly desirable if red giant models could predict $T_{\text {eff }}$ accurately, since it would allow better mass determinations for giants, and would remove large part of the age uncertainties in population synthesis for old galaxies. $T_{\text {eff }}$ depends on the opacities, the superadiabatic convection and the atmospheric boundary condition, which are all not known well enough. D. VandenBerg [12] concluded that it is impossible to fit effective temperatures on MS and RGB [of metal-poor globular clusters] simultaneously, and comparison between model predictions and empirically determined $T_{\text {eff }}$ of clusters of all metallicities shows that both on the low- and high- $Z$ end there are severe discrepancies (see http://www.lorentzcenter.nl/lc/web/2009/324/Monday/Weiss.pdf).

\footnotetext{
${ }^{1}$ http://www.lorentzcenter.nl/lc/web/2009/324/info.php3?wsid=324
} 
Ageing Low Mass Stars: From Red Giants to White Dwarfs
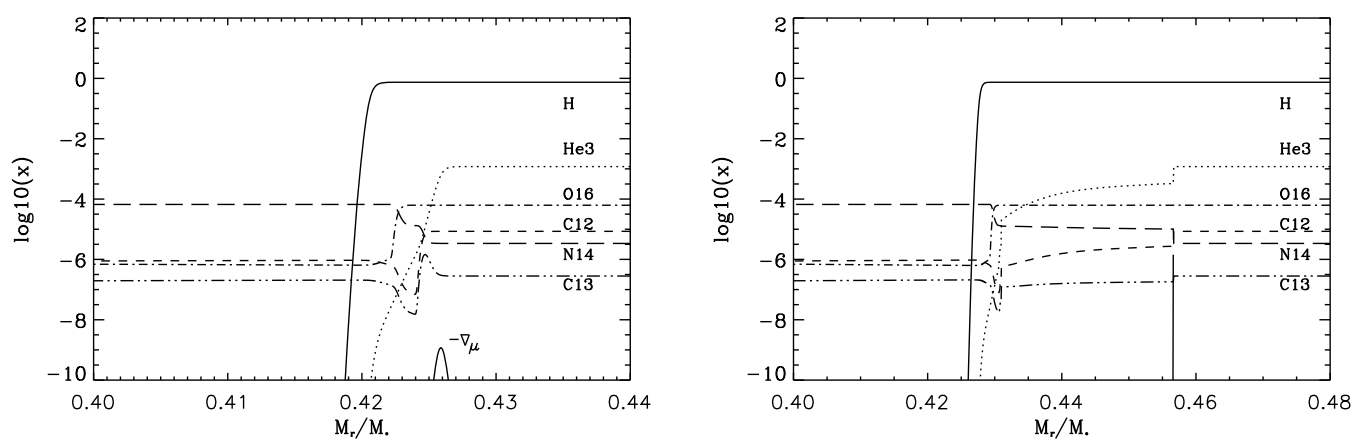

Figure 4. Results of models including fingering convection according to the results by [15]. Left: original efficiency of thermohaline mixing; right: efficiency enhanced by a factor of 1000 .

The same comparison also revealed that the luminosity of the RGB tip differs between codes by as much as $0.1-0.2 \mathrm{dex}$ in $\log L / \log L_{\odot}$, although some of the difference could be due to the numerical details of RGB calculations, and can be reduced. A systematic investigation was presented by Cassisi [13, and references therein], were it was also shown that for similar composition and physical assumptions the tip brightness difference between models can be reduced to the level of $0.1 \mathrm{mag}$, and that the helium core mass (intrinsically connected to the brightness) at the tip agrees within a few $10^{-3} M_{\odot}{ }^{2}{ }^{2}$ $\mathrm{He}$ also showed that the conductive opacities and the efficiency of diffusion (cf. Sect. 2.3) have the largest physical influence on the tip properties.

\subsection{Extra mixing on the RGB}

It is now common knowledge that there exists a mechanism in low-mass red giants that leads to mixing beyond the standard first dredge-up, after the star has reached the bump. As a consequence the ${ }^{12} \mathrm{C} /{ }^{13} \mathrm{C}$ ratio drops from $\approx 20$ to values around 10 , carbon depletes, and nitrogen gets enhanced, since CNOprocessed material is transported from the outer regions of the hydrogen-burning shell to the bottom of the convective envelope. Also, ${ }^{3} \mathrm{He}$, which was enhanced in the envelope during the MS, gets reduced again. While the way the extra-mixing works is well understood, the actual mechanism is still not known. Presently, thermohaline mixing (or, fingering convection) is the preferred explanation $[14$, see also the contribution by Lagarde, this volume], which is triggered by a very small molecular gradient inversion $\left(\delta \mu / \mu \approx-10^{-6}\right)$ caused by the ${ }^{3} \mathrm{He}\left({ }^{3} \mathrm{He}, 2 p\right)^{4} \mathrm{He}$ reaction in the outskirts of the shell. The modeling is done either by simplified one-dimensional approximations (with a free parameter), or by 3d-hydro models [15] (with a limited physical time covered). The hydro-results allow also to derive a 1d-mixing speed which can be implemented in a standard stellar evolution code.

Figure 4 shows our results implementing the recipe derived by [15] in the left panel. The $\mu$-inversion is located at $M_{r} / M=0.426$, and the effect on the composition is very moderate and restricted to the shell location. The right panel shows the result in case of an artificial (though not impossible, [15]) enhancement of the mixing speed by a factor 1000 . Now the profile is clearly modified and the element abundances changed in the right direction.

The next figure (Fig. 5) summarizes the effect on the surface abundances for several mixing attempts as function of age. While the original efficiency according to Traxler et al. [15] leads to no change at the surface (lower panel), the enhanced rate (middle panel) or the additional inclusion of overshooting from

${ }^{2}$ A. Bressan (private communication during this meeting) informed me that the new Padova models agree now at the same level with the other models shown in [13]. 
Figure 5. Surface element abundances (solid: C; dashed: N; dash-dotted: O) relative to their initial values, after thermohaline mixing was applied. Lower panel: original according to [15]; middle panel: enhanced by factor 1000 (as in Fig. 4, right panel); top panel: original plus additional overshooting. Models start at the bump (vertical dotted line) and are for $M=0.9 M_{\odot}, Z=10^{-4}$.

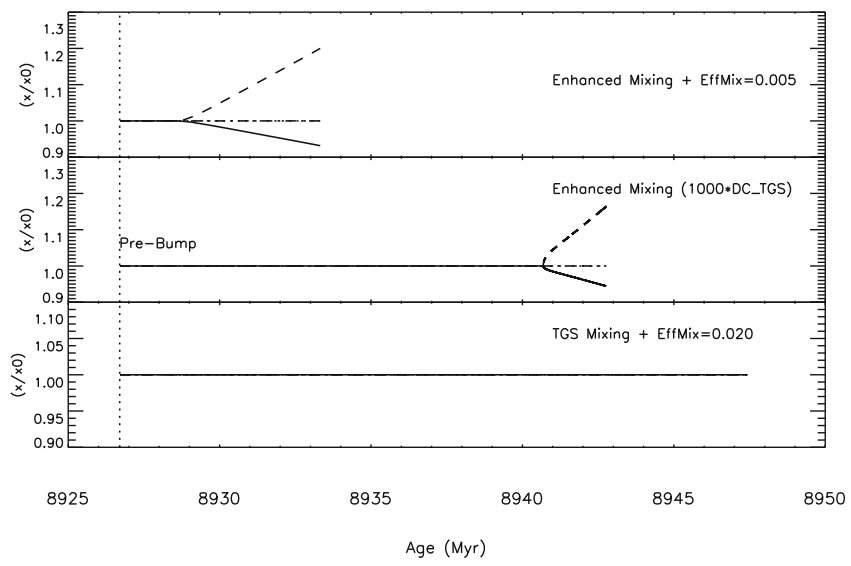

the convective envelope (top panel) lead to the desired effect. We close this subject by mentioning that also the alternative description by Kippenhahn et al. [16] leads to noticeable effects only when enhanced, and that generally hydro-models indicate a too low mixing efficiency [17]. The subject is thus still open.

\subsection{The location of the bump}

I already mentioned the bump, which shows up in luminosity functions of stellar clusters as an enhanced number of stars around $\log L / \log L_{\odot} \approx 2$, and which is due to the change in molecular weight, left over from the deepest extent of the convective envelope, when it is reached by the advancing hydrogenburning shell. It can, in principle, be used for distance and age determinations, for determination of the helium abundance, and more. However, its brightness appears to be inaccurately modeled. Apart from code-to-code differences found in the mentioned model comparison of the Leiden Giants meeting, it was also found repeatedly to disagree with observed locations in the HRD. Meissner \& Weiss [18] showed that (for their models) the bump location of GC-type isochrones was inconsistent with the TO brightness for any given age. The fact that the bump tends to be too bright by about 0.2 mag was confirmed, among others, by [13], in particular for the lowest metallicities.

There have been several possibilities proposed how to achieve a better agreement with the empirical bump brightness. What is needed is that the convective envelope extends deeper such that the composition discontinuity is reached at lower core mass - therefore luminosity - than in standard models. One way would be to enhance the opacities (relevant for the radiative gradient). We tested this but did not achieve the intended bump luminosity decrease even for significant opacity increase. The second option is overshooting from the convective envelope. This was investigated in [19], and the result is shown in Fig. 6. While diffusion tends to decrease the main-sequence duration, it has no influence on the bump brightness, in contrast to overshooting which increases lifetimes, but also decreases, as intended, the location of the bump by about $0.2 \mathrm{dex}$ in the HRD. Mass loss is of no influence. The overshooting parameter for this exercise was almost twice as large as needed for fitting open cluster CMDs, but the brightness reduction is $0.38 \mathrm{mag}$, which is also about twice the known discrepancy. Therefore, the "standard" overshooting parameter would probably be able to remove the discrepancy.

\subsection{The core helium flash}

I mentioned in the introduction that modern stellar evolution codes can follow the full core helium flash. However, these calculations are done under the assumption of hydrostatic equilibrium and with an insufficient treatment of convection: even if time-dependent mixing is assumed, the convection itself is 

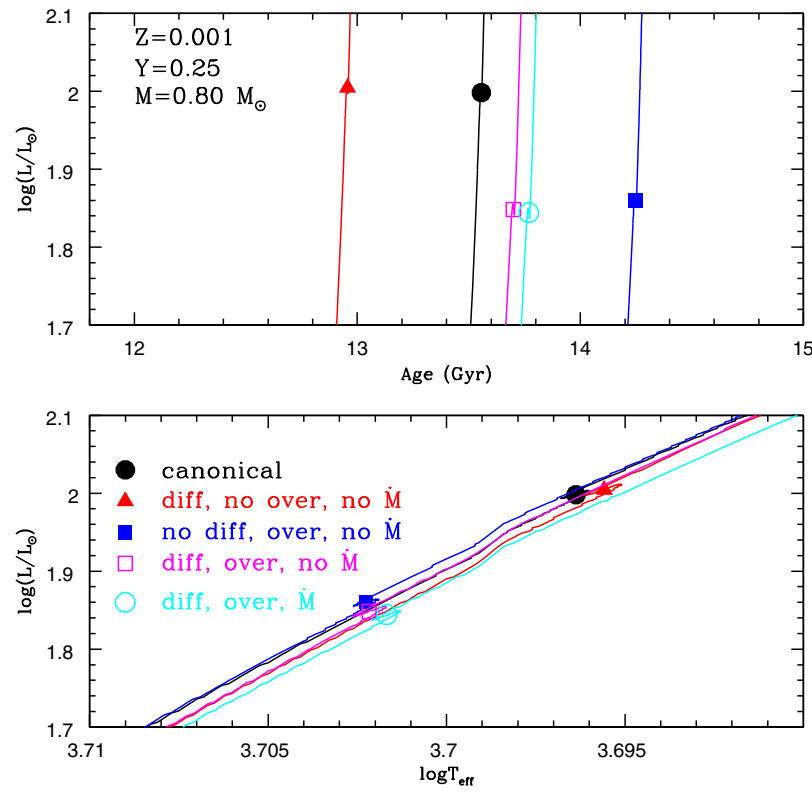

Figure 6. Sensitivity of the bump luminosity, in case of a model with $M=0.8 M_{\odot}$ and $Z=10^{-3}$, to different assumptions concerning the occurrence of diffusion, overshooting, and mass loss. Top: as function of age; bottom: location in HRD (from [19]).

still time-independent, i.e., is always assumed to be instantaneously adjusted to the very fast-changing thermal conditions in the flash. The complete luminosity evolution during the flash is shown in Fig. 7, from [20]). Note the appearance of so-called "minipulses" after the main flash, which are occurring during the heating and expansion of the core over a timescale of about 1 Myr. Stars experiencing such minipulses were identified to be - possibly - the theoretical counterpart of RR Lyr stars observed to have period changes of $|d \Pi / d t|>0.15 \mathrm{~d} / \mathrm{Myr}$ [21].

However, full 2d-and 3d-hydro simulations [22] indicate that the heating of the core occurs on a much shorter timescale (of order years or shorter) at a rate of $3000 \mathrm{~K} / \mathrm{s}$, due to convective entrainment. Consequently, the minipulses would vanish, and the mentioned RR Lyr stars have to be explained in a different way. Although these simulations could follow the evolution for only $36 \mathrm{hrs}$ of physical time, the results show that the physics of the core helium flash is far from full understanding. Another, still open question concerns the amount of mass loss during the flash. While it seems quite clear that mass loss on the RGB increases with luminosities, there is no consensus about the mechanism and detailed dependence on stellar parameters, and whether the flash plays an important role in this.

\section{THE HORIZONTAL BRANCH}

Whatever the details of the core helium flash are, and no matter how and when mass is lost: after the helium flash low-mass stars settle on the horizontal branch (or the clump, depending on their composition and mass) and experience a quiet phase of convective core helium burning. Due to the sensitivity of opacities - and thus of the radiative gradient-on composition the evolution of the convective regions is highly complicated, and depends a lot on the mixing prescription (instantaneous, time-dependent, composition-dependent), as well as on the stability criterion Schwarzschild or Ledoux). Without any overshooting, a discontinuity in the radiative gradient develops at the edge of the convective core, which thus will not grow in size (Fig. 8, left panel). If any, even small amount of overshooting (or growth of the core, which can be due to the technical treatment of convective boundaries) is allowed, $\nabla_{\text {rad }}$ develops a minimum within the core. Due to an overall reduction of $\nabla_{\text {rad }}$ this minimum at later times falls below the almost constant $\nabla_{\mathrm{ad}}$, stopping any convective mixing in a region inside the core 
Figure 7. Total, He-, and H-luminosity during the core helium flash of an $0.85 M_{\odot}, Z=10^{-3}$ stellar model [20]. Note the different timescales during the flash.
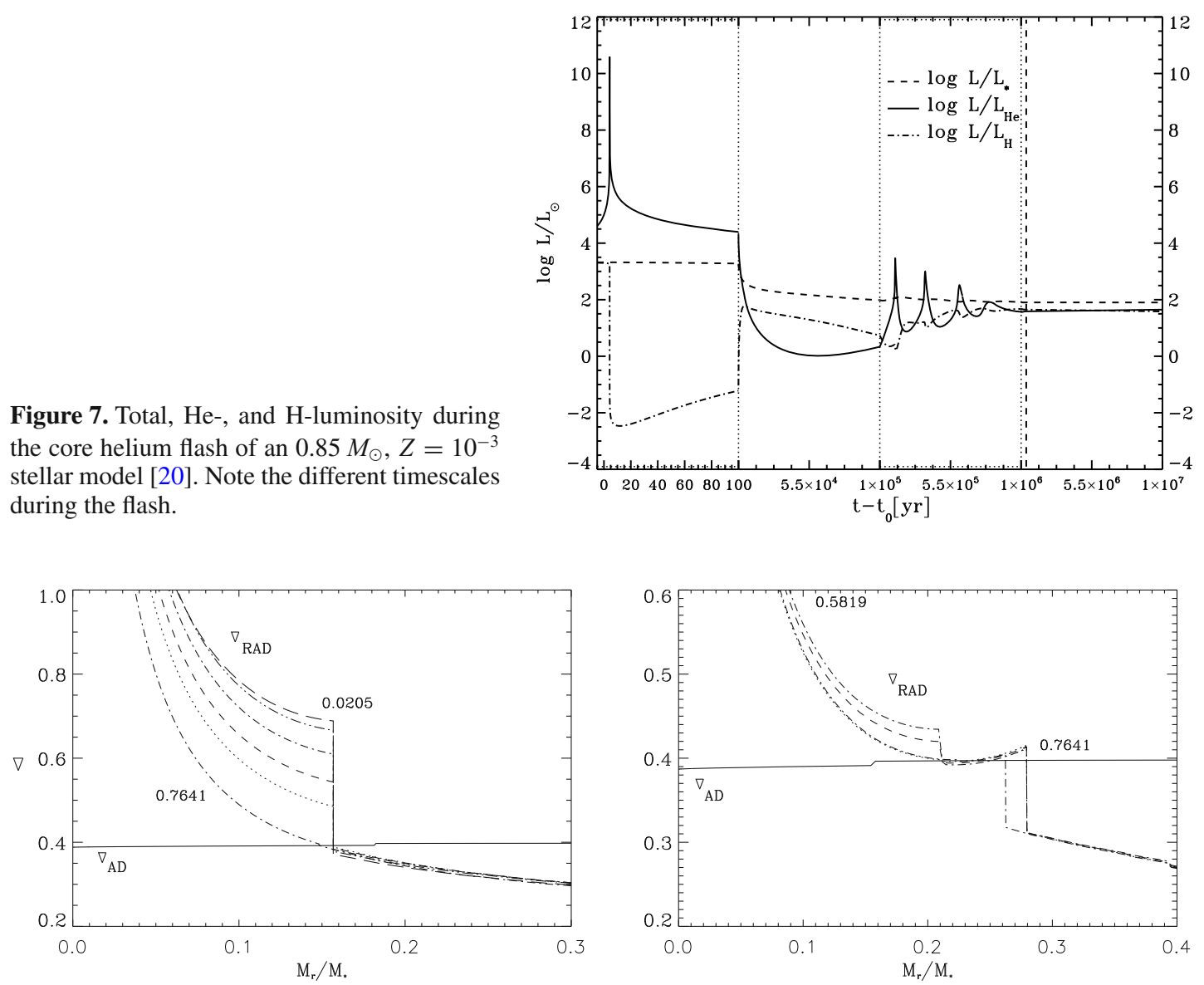

Figure 8. Gradients $\left(\nabla_{\mathrm{ad}}\right.$ and $\left.\nabla_{\mathrm{rad}}\right)$ during horizontal evolution of a star of $0.8 M_{\odot}\left(Z=10^{-4}\right)$. The numbers correspond to the central helium content of the various models shown by the different linestyles. Left: evolution without any core growth (Schwarzschild-criterion for convection); right: core growth due to overshooting (Ledouxcriterion).

immediately. However, some mixing would make the region unstable again, and we have a situation sometimes called "semiconvective", although I prefer "semi-mixing". In our case, due to the use of the Ledoux-criterion and the inclusion of a small amount of time-dependent mixing by overshooting a chemical gradient is established that leads to marginal stability in the critical region, as can be seen in the right panel of Fig. 8. In some codes the mixing is done in an ad-hoc manner with the aim of reaching exactly this marginal stability situation.

The further evolution on and after the HB depends on the details of the chemical profile established during core helium burning. In particular it determines how fast and at which luminosity the RR Lyr instability strip is crossed. At the end of core helium burning the so-called breathing pulses appear in many calculations. These are sudden extensions of the convective core size, mixing fresh helium into the burning region, rejuvenating thus the core. They are believed to be mostly a numerical problem, but their occurrence also depends on details of the treatment of convection. Finally, it should be mentioned that the ${ }^{12} \mathrm{C}(\alpha, \gamma){ }^{16} \mathrm{O}$-reaction rate is still a significant source of uncertainty for the $\mathrm{HB}$ evolution, but also for later phases, in particular for the white dwarf cooling phase, as it determines the C/O-ratio in the core. 


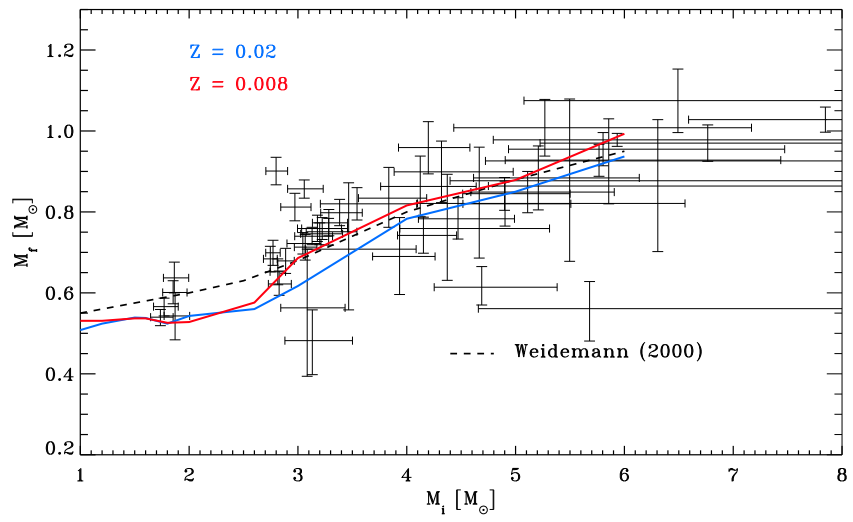

Figure 9. Initial-final mass relation deduced from observations [30] and the theoretical prediction from our models [29] for two different metallicities. The classical IFMR by Weidemann [31] is shown for comparison.

\section{THE ASYMPTOTIC GIANT BRANCH PHASE}

The AGB is the final culmination of all problems mentioned so far. It is thus no wonder that details of the evolution on the AGB differ significantly from author to author, as can easily be verified in the literature. We mention here only the work by A. Kitsikis [23], who finds for example, that already the core mass at the first thermal pulse differs widely from those published in [24] for masses in the transition range between low- and intermediate-mass stars (these are the two most extensive recent sets of AGB models), while it agrees well with models by M. Miller Bertolami (private communication). Similar discrepancies exist in the lifetimes on the AGB and other issues. Some of these problems may arise from the different strategies to achieve the third dredge-up, the detailed mechanism of which is still not understood. Kitsikis (and ourselves) use overshooting from all convective boundaries to achieve the enrichment of the envelope with carbon and the generation of the ${ }^{13} \mathrm{C}$-pocket, needed for the onset of s-processing.

The occurrence and amount of overshooting at the various convective boundaries (envelope, intershell convection zone) is highly disputed: to achieve the composition of PG1159 stars, [25] postulates a typical value at the bottom of the intershell convection zone, while in [26] "half of typical" is claimed for the production of typical s-process abundance pattern. On the other hand, "several times the typical value" for overshooting from the bottom of the convective envelope is needed for an efficient ${ }^{13} \mathrm{C}$ neutron source [27], and generally, in 3d-hydro simulations a variation by a factor of 10 is found [28].

Overshooting (from the bottom of the intershell convection zone) is also influencing the growth of the core. In our calculations it is actually preventing the core to grow beyond its size at the first TP for stars below $3 M_{\odot}$ [29]. As shown in Fig. 9, our theoretically predicted initial-final-mass-relation falls below the measured white dwarf masses in this mass range, while overall there is quite good agreement.

We close the AGB evolution with the comment that all current calculations experience great convergence problems at the end of the AGB, when the transition to the post-AGB phase should set in. The physical reason is that radiation pressure is sufficient to balance gravity in some region of the convective envelope, which therefore becomes unbound, and a radiation-hydrodynamical treatment would be warranted. The underlying reason might be the more pronounced "metal-bump" in the up-todate Rosseland mean opacities.

\section{CONCLUSION}

Although at first look simple structures, low-mass stars still pose many open questions and a number of unresolved problems to stellar modelers. Many of these are connected to the treatment of convection, either to the details of superadiabatic convection (as on the RGB, Sect. 3.1, and AGB, Sect. 5) or to overshooting from the various convective regions (from the PMS, Sect. 2.1, to the AGB, Sect. 5). 
While detailed analysis of observable quantities, which depend on convective overshooting, helps in some cases to "calibrate" its efficiency in certain circumstances (as in Sect. 2.2), an ab-initio understanding is what is really needed. All uncertainties accumulate during the evolution and become most evident on the AGB. It therefore is no surprise that models differ the most in this evolutionary phase. Nevertheless, already on the main-sequence, surprising and disturbing discrepancies between the output of different stellar evolution codes are found (Sect. 2.3). It is therefore clear that there is still a lot of room for improvements, and that these improvements are urgently needed, in order to provide the asteroseismology community with accurate low-mass stellar models.

It is a pleasure to thank the organizers for the invitation and for their financial support. This work was also supported by the DFG cluster of excellence "Origin and Structure of the Universe" (www . universe -cluster .de).

\section{References}

[1] A. Weiss, H. Schlattl, Astrophys. Space Sci. 316, 99 (2008)

[2] B. Ahrens, M. Stix, M. Thorn, Astron. Astrophys. 264, 673 (1992)

[3] P. Molaro, A. Bressan, M. Barbieri, P. Marigo, S. Zaggia, Mem. Soc. Astron. It. Suppl. 22, 233 (2012)

[4] H. Schlattl, H., A. Weiss, Astron. Astrophys. 347, 272 (1999)

[5] G. Wuchterl, R. Klessen, Astrophys. J. 560, L185 (2001)

[6] I.-J. Sackmann, A.I. Boothroyd, Astrophys. J. 583, 1024 (2003)

[7] R. Rye, H.K. Phillip, H.D. Holland, Nature 378, 603 (1995)

[8] M. Asplund, N. Grevesse, A.J. Sauval, P. Scott, Ann. Rev. Astron. Astophys. 47, 481 (2009)

[9] D.A. VandenBerg, B. Gustafsson, B. Edvardsson, K. Eriksson, J. Ferguson, Astrophys. J. 666, L105 (2007)

[10] Z. Magic, A. Serenelli, A. Weiss, B. Chaboyer, Astrophys. J. 718, 1378 (2010)

[11] M. Marta, et al., Phys. Rev. C 78, 022802 (2008)

[12] D.A. VandenBerg, Phys. Scr. T133, 014026 (2008)

[13] S. Cassisi, Red Giants as Probes of the Structure and Evolution of the Milky Way (Springer, Berlin Heidelberg), p. 56 (2012)

[14] C. Charbonnel, J.-P. Zahn, Astron. Astrophys. 467, L15 (2007)

[15] A. Traxler, P. Garaud, S. Stellmach, Astrophys. J. 728, L29 (2011)

[16] R. Kippenhahn, G. Ruschenplatt, H.-C. Thomas H.-C., Astron. Astophys. 91, 17 (1980)

[17] P.A. Denissenkov, Astrophys. J. 723, 563 (2010)

[18] F. Meissner, A. Weiss, Astron. Astrophys. 456, 1085 (2006)

[19] F. Troisi, et al., Pub. Astr. Soc. Pacific 123, 879 (2011)

[20] A. Serenelli, A. Weiss, Astron. Astophys. 442, 1041 (2005)

[21] V. Silva Aquirre, M. Catelan, A. Weiss, A.A.R. Valcace, Astron. Astophys. 489, 1201 (2008)

[22] M. Mocak, E. Müller, A. Weiss, K. Kifonidis, Astron. Astophys. 501, 659 (2009)

[23] A. Kitsikis, PhD (Techn. Univ. Munich), (2008)

[24] A.I. Karakas, PhD (Monash University, Melbourne), (2003)

[25] F. Herwig, T. Blöcker, N. Langer, T. Driebe, Astron. Astophys. 349, L5 (1999)

[26] M. Lugaro, F. Herwig, J.C. Lattanzio, R. Gallino, O. Straniero, Astrophys. J. 586, 1305 (2003)

[27] F. Herwig, N. Langer, M. Lugaro, Astrophys. J. 593, 1056 (2003)

[28] F. Herwig, et al., ASP Conference Series 378, p. 43 (2007)

[29] A. Weiss, J.W. Ferguson, Astron. Astophys. 508, 1343 (2009)

[30] M. Salaris, A. Serenelli, A. Weiss, M.M. Miller Bertolami, Astrophys. J. 692, 1013 (2009)

[31] V. Weidemann, Astron. Astophys. 363, 647 (2000) 